\title{
HOLISM ABOUT VALUE: SOME HELP FOR INVARIABILISTS
}

\author{
Author Name: Daniel Halliday
}

Affiliation: School of Historical and Philosophical Studies, University of

Melbourne, VIC 3010, Australia

Email: daniel.halliday@unimelb.edu.au

Tel:

Fax:

\begin{abstract}
:
G.E.Moore's Principle of Organic Unity holds that the intrinsic value of a whole may differ from the sum of the intrinsic values of its parts. Moore combined this principle with invariabilism about intrinsic value: An item's intrinsic value depends solely on its bearer's intrinsic properties, not on which wholes it has membership of. It is often said that invariabilism ought to be rejected in favour of what might be called 'conditionalism' about intrinsic value. This paper is an attempt to show how invariabilism might be filled out in ways that allow its proponents to answer their conditionalist opponents. The main point consists in identifying how some amount of extrinsic part-value may contribute to whole-value that is nevertheless intrinsic. This enables an invariabilist to explain how the intrinsic value of a whole may differ from the sum of its intrinsic part-values, without abandoning the Moorean doctrine that intrinsic value supervenes on intrinsic properties (the proposal is nevertheless consistent with the view that invariabilist and conditionalist accounts might exist side by side). I finish with a brief explanation of how the main proposal could help construct invariabilist accounts of particular organic unities, looking beyond the more general argument they have with conditionalists.
\end{abstract}

Keywords: Moore; Holism; Intrinsic Value; Extrinsic Value; Invariabilism; Organic Unity 


\section{Daniel Halliday}

\section{Introduction}

In 1903, G.E.Moore wrote that "the value of a whole must not be assumed to be the same as the sum of the values of its parts"1. This claim is commonly known as his Principle of Organic Unity. Moore's principle is certainly a very plausible one. At least, candidate 'organic unities' (the sort of 'wholes' to which Moore's principle refers) seem quite easy to think of: A mosaic gets its value not merely from the value of each of its stones, but (it might be said) from the way they are arranged. Jumble up the stones a bit, and you are likely to spoil the mosaic. But you won't have made any of the stones worse. Similarly, pleasure seems like a good thing and malice a bad thing. How should we evaluate the pleasure that a sadist gets from witnessing another person's undeserved misery? Well, it seems that we can't simply adjust the positive value of the sadist's pleasure by subtracting the disvalue of her pernicious character. This would be too much like weighing these factors as if they had occurred, unrelated, in separate individuals. These sorts of considerations account for the enduring interest in Moore's claims.

A ready supply of cases like those above supports the truth of Moore's principle ${ }^{2}$. However, cases alone do not immediately point towards any particular explanation of its truth. The Principle of Organic Unity is, on its own, a merely negative claim: It leaves much open as to what would be included in a fuller, positive account of what valuable organic unities are like. Providing such an account requires a theory of holism about value ${ }^{3}$. In what follows, I shall

\footnotetext{
${ }^{1}$ (1903: 28).

${ }^{2}$ Moore's principle is not accepted by everyone. Michael Zimmerman $(1999,2001)$ has argued that that the bearers of intrinsic value are states of objects, rather than objects themselves (or facts, or states of affairs). Very roughly, Zimmerman's view implies that items do not bear intrinsic value in isolation from complicated states in which they are found (rendering empty any principle about summing such values). A proper engagement with Zimmernan's views would require an extended discussion of what the bearers of intrinsic value might be, for which there is insufficient space (although see note 27, below). In any case, this problem lies outside my focus on overcoming objections made by conditionalist opponents of invariabilism.

${ }^{3}$ Strictly speaking, value holism actually rules out more than just ideas about summing the values of parts. Moore's writings fail to make this obvious. Really, holism contrasts with value atomism. Denying atomism is actually to make a stronger claim than denying any principle about summing. The contrast between atomism and holism is highlighted by Brown (2007: 457-459) and Dancy (2004: 94-95). A good background discussion of atomism can be found in Broome (1991: Ch.4).
} 
present some arguments that support 'invariabilist' explanations of organic unities. I will aim to show, principally, how an invariabilist may secure some of the advantages that are often said to be available only on rival accounts of holism about value. To do this, I shall explain how an intrinsically valuable whole may confer extrinsic value on its parts (or, alternatively, that parts within a whole may confer such value on each other). This leads to the view that the intrinsic value of a whole may be to some extent constructed out of non-intrinsic value of its parts. The paper's argument is not, I should add, intended to support the more general view that invariabilism provides the right account of all organic unities, and that alternative accounts have no application. Rather, my aim is to help invariabilists respond to the charge that their view has no application, which is something that proponents of non-invariabilist accounts have often suggested.

\section{Conditionalism versus Invariabilism}

Let me say a few things about what distinguishes invariabilist accounts of organic unity from their main rival. (To make the distinction clear, it will be convenient to talk of these two accounts as if they are supposed to be fully general.)

Invariabilists believe that an item's intrinsic value cannot vary without some variation in the intrinsic nature of that item. This idea is often expressed in terms of intrinsic value supervening on its bearer's intrinsic properties. Being a member of a whole is not an intrinsic property ${ }^{4}$. So, properties of membership cannot (for invariabilists) be relevant to intrinsic value. Moore was an invariabilist himself, writing that "the part of a valuable whole retains exactly the same value when it is, as when it is not, a part of that whole" ${ }^{\prime 5}$ Invariabilism can be contrasted with a view that permits a certain sort of flexibility about intrinsic value. We can call this view, 'conditionalism' about intrinsic value ${ }^{6}$. Conditionalists believe that the intrinsic value of an item

\footnotetext{
${ }^{4}$ See Noah Lemos's presentation of what he calls the "Principle of Universality" (1994: 11). Dancy refers to the same view as the "local doctrine of supervenience for intrinsic value" (2003: 631). (Membership of a whole might be an intrinsic property when an item is a member of itself, but such cases may be set aside.)

5 (1903: 30).

${ }^{6}$ I should say that I am forced to be selective in using these labels, since there is not perfect consistency across the literature. Dancy sometimes uses “variabilism” to refer to what I'm calling 'conditionalism'. Thomas Hurka (1998) uses "holism" to label what I have called 'invariabilism', although I, like others, prefer to think of both views as alternative conceptions of holism.
} 
may change when it has membership of a relevant whole: An item might have more or less intrinsic value than when isolated, or when part of some other whole. Jonathan Dancy, for example, claims that parts may "change their value as they move from whole to whole"7.

Conditionalist accounts require us to give up the Moorean idea that intrinsic value can't change without some change in its bearer's intrinsic properties. This may seem like a cost. Invariabilists, at any rate, are motivated partly by the intuition that an item's intrinsic value is a matter of what that thing is like in itself, and not how it is related to other things ${ }^{8}$. In return, however, conditionalism offers an attractively straightforward account of how organic unities occur. Invariabilists, by contrast, seem to be left with a certain burden of explanation not shared by their conditionalist opponents: If intrinsic value can't vary in the absence of any variation in its bearer's intrinsic properties, then how can organic unities occur in the first place? If valuable parts can combine to form valuable wholes, but don't thereby undergo a change in intrinsic value, then why isn't the intrinsic value of the whole equal to the sum of its parts' intrinsic values? Invariabilists need to explain what further feature of organic unities allows their intrinsic value to be greater or lesser than the sum of parts' values ${ }^{9}$. Holding on to Moorean ideas about supervenience makes it look harder for them to do this.

In order to meet the challenge posed above, invariabilists might adopt either of the following two principles:

The Relations Principle: The intrinsic values of relations between a whole's parts contribute to the intrinsic value of that whole.

The Non-Dispersion Principle: The non-intrinsic (and non-instrumental) values of a whole's parts contribute to the intrinsic value of that whole.

\footnotetext{
7 (2003: 637, 629). A similar phrasing appears in Lemos (1994: 33). Korsgaard (1983) is another prominent defence of conditionalism.

${ }^{8}$ Insofar as this paper is an attempt to improve invariabilism, it is permissible to assume that it's worth retaining the view that intrinsic value does supervene upon intrinsic properties. I should note, though, that it finds opposition in Dancy (2003: 632-33, 2004: 38-41), Dorsey (2012), and Kagan (1998). Bradley (2002) defends Moorean supervenience against some of these authors' objections.

${ }^{9}$ I should say that the question of whether to adopt invariabilism or conditionalism needn't turn entirely on which view give the best account of organic unities. Conditionalists may also draw support from certain claims about the nature of intrinsic value, which contrast with some claims made by Moore. For more on Moorean vs Kantian conceptions of intrinsic value, see Bradley (2006).
} 
Both principles say something about the calculation of a whole's intrinsic value, accounting for why that value could be greater or less than the sum of the intrinsic part-values. The relations principle achieves this by identifying another bearer of intrinsic value, namely, certain relations between parts. Relations between parts don't exist 'in isolation' from the relevant whole. So, the relations between a whole's parts wouldn't be included if one were to sum the intrinsic values held by respective parts, construed in isolation from the whole ${ }^{10}$. The non-dispersion principle works in a different way. Instead of identifying a further bearer of intrinsic value, it denies that intrinsic whole-value must be assembled entirely out of intrinsic part-values. It asserts that some amount of part-value may be non-intrinsic, and yet (crucially) still contribute to whole-value that is intrinsic. The idea here is that the intrinsicness of whole value need not 'disperse' into partvalue that is entirely intrinsic. The non-dispersion principle is rather less straightforward than the relations principle. However, as I shall argue, invariabilists might be well advised to make room for it.

This paper is motivated partly by the sense that invariabilists have not said much about either the relations principle or the non-dispersion principle. At any rate, no such distinction is really highlighted in the literature. But invariabilists ought to say something about these principles. After all, they need to explain why it is that intrinsic whole-value differs from the sum of intrinsic part-values. And as part of providing this explanation, invariabilists need to identify some input in the calculation of whole-value that is not an intrinsic part-value. The relations principle and the non-dispersion principle offer two distinct ways of identifying this other input. So invariabilists should ask themselves which principle they prefer, even if they might switch between these principles when accounting for different organic unities ${ }^{11}$.

\footnotetext{
${ }^{10}$ These remarks allow that a relation between parts of a whole is itself a part of that whole. But it is not the sort of part that can be isolated for the purposes of summing the intrinsic values that parts hold in isolation.

${ }^{11}$ I should add that the relations principle and the non-dispersion principle are not exhaustive of invariabilist logical space, at least if the Moorean supervenience claim is regarded as the essential component of invariabilism. It is possible that the intrinsic value of a whole could supervene on its intrinsic properties, even if the parts of that whole (and the relations between them) were wholly valueless. Any way of accounting for such an organic unity could not draw on the relations principle or the non-dispersion principle. I thank an anonymous reviewer for pointing this out to me.
} 
I should say that the literature on organic unity isn't wholly devoid of any awareness of the relations and non-dispersion principles. At least, some contemporary invariabilists seem to presuppose the relations principle in their formulations ${ }^{12}$. And the non-dispersion principle is sometimes gestured at ${ }^{13}$. On the whole, invariabilists have not thought carefully about what might contribute to intrinsic whole-value, other than intrinsic part-value. Until such a task is attempted, however, invariabilists are less able to explain organic unities than conditionalists. Moore, it is worth noting, probably didn't accept the relations principle. At least, there is no textual evidence establishing that he thought relations between parts could bear intrinsic value. On the other hand, Moore didn't recognise any sort of non-intrinsic value other than instrumental value. This suggests he would have rejected the non-dispersion principle, which relies on the possibility of extrinsic non-instrumental value. That said, most authors nowadays take Moore to have been wrong on the second point, and it's quite possible he was wrong on the first as well.

In what follows, I shall offer some reasons why invariabilists might do better to accept the non-dispersion principle rather than the relations principle. My arguments do not depend on whether existing invariabilists have in fact tended to accept the relations principle instead, or whether invariabilists have in fact said things wholly consistent with the non-dispersion principle. If in fact they have, then so much the better. In demonstrating the advantages of the non-dispersion principle, I will mainly aim to construct responses to claims that conditionalists make against invariabilism in general. As such, my main arguments will not include many claims about any particular cases of alleged organic unities. However, readers may also find it helpful to see how the non-dispersion principle may be put to work in invariabilist accounts of particular organic unities. So, I will spend some time towards the end of the paper examining how the nondispersion principle compares with the relations principle in giving an account of cases of malicious pleasure, which are commonly discussed in connection with Moore's principle. I do not claim, ultimately, that the relations principle has no role to play in invariabilist accounts of

\footnotetext{
${ }^{12}$ Thomas Hurka describes invariabilism like this: "The intrinsic value in a whole composed of two or more parts standing in certain relations need not equal the sum of the intrinsic values those parts would have if they existed alone, or apart from those relations" (1998: 300). Hurka goes on to say that invariabilists "value relations" (307) and that these may be causal relations between the various parts of a whole (302).

${ }^{13}$ Ben Bradley suggests that an item can have value "in virtue of being a part of a valuable whole" (1998: 110). Bradley identifies this sort of value as non-instrumental extrinsic value, which makes his view close to an affirmation of the non-dispersion principle. See also the remarks from Chisholm, below.
} 
organic unity. But I am concerned to show that invariabilists have good reasons to make significant use of the non-dispersion principle.

\section{Dispersion and Evaluative Priority}

As I have said, the non-dispersion principle is a little less straightforward than the relations principle. In this section, I will concentrate on spelling out the relevant idea of 'nondispersion'. Speaking rather abstractly, 'dispersion' pertains to the idea that some property of a whole is also a property of its different parts. Setting aside matters about value for a moment, dispersion can be first explained in terms of more ordinary intrinsic properties. Think, for example, of the statue of Abraham Lincoln that sits in the Lincoln Memorial in Washington, DC. This statue has the property of being made of Georgia Marble. Since the statue is made of Georgia Marble and nothing else, let's say that its parts are made of Georgia Marble ${ }^{14}$. Here we have an example where an intrinsic property of some whole is also an intrinsic property of its parts: Being made of Georgia Marble is an intrinsic property that disperses in the sense I have in mind. Not all intrinsic properties disperse in this sense. For example, the statue of Lincoln also bears the intrinsic property of having shorter arms than legs. But this property is not held by all of the statue's parts ${ }^{15}$. Lincoln's arms of course have the property of being shorter than his legs, but that is an extrinsic, relational property, since Lincoln's arms have that property conditional on facts about what is external to his arms (the same goes for Lincoln's legs having the property of being longer than his arms).

When intrinsic whole-properties do not disperse, they often stand in the same relations to part-properties as when they do disperse. At least, certain sorts of inferences remain available irrespective of whether an intrinsic property disperses among a whole's parts, or whether those

\footnotetext{
${ }^{14}$ This is not strictly true: As a referee has pointed out, the statue may have (say) carbon atoms among its parts, and these aren't made of Georgia Marble. I suspect that it is possible to overcome this complication like this: Some properties disperse insofar as they are held by all of an object's parts that are above some threshold separating (stipulated) macro- and micro-levels, and held by no parts identified as falling below this threshold. We may still contrast this with other properties fail to disperse insofar as they are held by some but not all parts at a given level. This modification allows us to distinguish the statue's property of being made of Georgia Marble (which is held by all of its parts down to, we might say, its constituent calcium carbonate molecules) and the property of having ears, which is held by some parts at the macro-level, but not other parts at that level (see following footnote).

${ }^{15}$ It is perhaps held by some proper parts, such as the statue minus its head. Of course, some intrinsic properties don't disperse: Lincoln's statue has ears, but his left foot doesn't have ears.
} 
parts happen to bear only extrinsic properties. From the fact that Lincoln's parts are of Georgia Marble, one can infer that the statue itself is of Georgia marble. Similarly, Lincoln's having the intrinsic property of shorter legs than arms is something we can deduce from his arms' having the extrinsic property of being shorter than his legs. Knowing about the properties of a whole's parts normally permits certain inferences about the intrinsic properties of that whole. But these inferences are not only available on knowledge of parts' intrinsic properties alone. Indeed, if we only drew the inferences about intrinsic whole-properties that could be drawn from intrinsic partproperties, then we'd be liable to gain an incomplete list of whole-properties. As the example of Lincoln's statue indicates, we can often infer facts about a whole's intrinsic properties from facts about its parts' extrinsic properties.

What I've called 'dispersion' enjoys a more general sort of plausibility. This is often noticed by philosophers interested in the metaphysics of properties. David Lewis, for example, explains that what's intrinsic to a whole may be merely extrinsic to its parts:

\footnotetext{
An ascription of extrinsic properties to something is not entirely about that thing, though it may well be about some larger whole which includes that thing as part. A thing has its intrinsic properties in virtue of the way that thing itself, and nothing else, is. Not so for extrinsic properties, though a thing may well have these in virtue of the way some larger whole [intrinsically] is."16
}

The points I have made about Lincoln's statue are really just a way of demonstrating what Lewis is pointing out in these remarks: Generally, we should not always seek to reduce the intrinsic nature of a whole to the intrinsic nature of its parts, even though we could still learn something about that whole by first learning about extrinsic features of its parts. We should keep in mind the possibility that valid inferences from part-facts to whole-facts do not establish any metaphysically stronger sort of priority that parts have over their wholes. The non-dispersion principle is one way of refining this idea with respect to evaluative priority in particular.

When a whole's intrinsic value is greater or less than the sum of its parts' intrinsic values, this may be because some amount of intrinsic whole-value obtains, whose presence can't be inferred from the presence of any intrinsic part-value (or disvalue). In such a case, the lesson of

${ }^{16}$ (1983: 111-112, italics added) 
Moore's principle of organic unity is that summing intrinsic part-value may leave out information relevant to an accurate calculation of intrinsic whole-value. In fact, to say this is just to repeat the claims I've made for non-value properties. The non-dispersion principle (for value) will be more persuasive, however, if some explanation can be given of why intrinsic whole-value doesn't always disperse. I shall now suggest that this has to do with the way in which a whole might confer value on its parts, rather than (say) the other way round. I shall do this by drawing on some plausible claims that others have made about the way in which items can come to have extrinsic value.

Following Rae Langton, we may regard extrinsic value as falling into two categories the sort gained when an item has intrinsically valuable effects, and the sort gained when an item has value conferred on it from some distinct but intrinsically valuable source. In the second case, an item gains value from some source in ways that are non-causal ${ }^{17}$. This second, non-causal category of value is the sort of extrinsic value that is relevant to the defence of the nondispersion principle. The fact that extrinsic value is conferred by an external source means that it fails to supervene on its bearer's intrinsic properties, thereby remaining distinct from intrinsic value as well as from the instrumental sort of extrinsic value. It's worth noting that an item can be extrinsically valuable and still be something that someone values intrinsically. In other words, the fact that an item gets its value from elsewhere does not mean that it can't be valued for its own sake, or as an end ${ }^{18}$. We may, for example, come to value something intrinsically because it has contingently come to represent something of intrinsic value ${ }^{19}$.

These claims about extrinsic value provide an account of an organic unity consistent with invariabilism about intrinsic value, but also consistent with the rejection of the relations principle. We have been led to the claim that contributive value need not be wholly intrinsic, even when the value it contributes to is wholly intrinsic. This idea can be restated in the following proposal: A sufficient condition for an organic unity is that non-instrumental extrinsic

17 (2007). Langton's discussion builds on (and somewhat revises) the well-known taxonomy in Korsgaard (1983). There are further concepts relevant to Langton's taxonomy, such as 'final' value, which I lack the space to discuss. Bradley (1998) identifies some additional categories as well. My use of categories such as 'intrinsic', 'extrinsic', and so on is guided by principally by Langton's discussion, rather than by any competing views in the literature. This is not to say that Langton would be committed to my own conclusions.

${ }^{18}$ For a fuller defence of this claim, see Rabinowicz \& Ronnow-Rasmussen (1999). See also Langton (2007: fn 10).

${ }^{19}$ Langton suggests the case of a wedding ring as an example of an item that could play this role. 
values of a whole's parts contribute to the intrinsic value of that whole. This results in the intrinsic value of that whole being greater or lesser than the contribution made by the sum of the intrinsic values of its parts.

It bears emphasising, here, that Moore's actual Principle of Organic Unity makes no claim about evaluative priority. As such, it presents no obstacle to accounts of value-holism that make use of ideas about evaluative priority. All Moore's principle is committed to is the absence of a valid inference from the sum of the intrinsic values of a whole's parts, to the amount of intrinsic value attaching to a whole itself. This says nothing about whether the parts being valuable makes it the case that the whole is valuable. Rather, the sense of 'contribution' of value is one that alludes only to the idea that part-value may ground some sort of inference about an amount of whole-value. The intrinsic value of a whole may remain an ultimate source of some amount of part-value, notwithstanding the capacity of parts to have intrinsic value when in isolation $^{20}$. Appreciating this point is an important element of seeing how the above proposal could be accepted by an invariabilist about intrinsic value.

\section{Meeting some objections to invariabilism about intrinsic value}

Some opponents of invariabilism seem to have treated Moore's principle of organic unity as something stronger than a point about what can't be reliably inferred from information about intrinsic part-value. This can be found in Jonathan Dancy's rejection of the view that parts could accrue non-instrumental extrinsic value when they join intrinsically valuable wholes ${ }^{21}$. Dancy construes this possibility as "parts contributing more value than they themselves have". He sees this as incoherent. More precisely, Dancy worries that such a view affirms a presence of partvalue without the presence of the reasons governing our treatment of that part:

\footnotetext{
We should be wary of the idea that a part can contribute more value to a whole than it has actually got there...[because] such a claim severs the connection between values and reasons... The general idea is that where there is value, there are reasons of certain sorts - reasons to cherish, respect, tend, approve, defend, and so on. So to say that a part with no value can contribute value that it
}

\footnotetext{
${ }^{20}$ This is roughly what Bradly refers to as a whole's "basic intrinsic value". This he describes as "value not derived from...parts, but instead...from the way the parts are put together" (2002: 34). Bradley attributes this view to Moore as well. It sounds to me like a denial of the view that parts have evaluative priority over a whole they're in. ${ }^{21}$ (2003: 633-34).
} 
has not got commits one to saying, it seems, that though there is no reason to preserve the part as a part, there is a reason to protect the whole, and that reason derives from the presence of the part.

So to say that a part with no value can contribute value that it has not got commits one to saying, it seems, that though there is no reason to protect the part as a part, there is a reason to protect the whole, and that reason derives from the presence of the part. Now this does sound incoherent. Surely we do have reason to protect the part here, if it is contributing value. ${ }^{22}$

The argument of this passage relies on the claim that intrinsic whole-value derives wholly from intrinsic part-value. If this claim were true, then there would indeed be something puzzling about the claim that we have reason to value a whole but no reason to value its parts. But the nondispersion principle allows this suppressed premise to be rejected. If intrinsic whole value is a sum of non-intrinsic part-values, then we could have reason to value the whole without any reason to value the parts as such. This point can be made more vivid if we imagine an organic unity that has intrinsic whole value but not intrinsic part value ${ }^{23}$. The Mona Lisa may have intrinsic value, even if its constitutive molecules do not. These molecules have extrinsic value, conferred on them by the intrinsic value of the whole painting. The Mona Lisa qualifies as an organic unity insofar as summing the intrinsic value of the molecules (which gives zero), does not yield the value of the painting (this being greater than zero). Now it's still true that the value of the Mona Lisa is in some sense "derived" from the presence of the molecules it comprises: The painting could not have its intrinsic value, were it not for its constituent molecules. These parts contribute extrinsic value that they have not "got" intrinsically, but only in the inferential sense of 'contribute' that pertains to the possibility of validly inferring facts about whole-value from facts about part-value. So, we can coherently say, after all, that we have reason to protect the Mona Lisa but no reason to value its molecules (except in a sense derivative of the reasons we have for valuing the whole painting). Thus, Dancy overlooks the idea that whole-value can be inferred from facts about part-value, but where these facts about part-value obtain partly because of facts about intrinsic whole-value. The view that contributive value must be intrinsic value might be plausible on the view that contributive value is really some kind of source of wholevalue. But on an inferential reading of 'contributive value', parts (or, for that matter, relations between parts) need not be evaluatively fundamental in this way. To deny this point is to simply

\footnotetext{
22 (2003: 630-31. Dancy repeats these claims at 2004: 177).

23 This suggestion, and the example of the Mona Lisa, are inspired by the comments of an anonymous reviewer (I should note that I have somewhat adapted the reviewer's suggested use of the example).
} 
stipulate that parts have evaluative priority. Once the commitment to parts as evaluatively fundamental is removed from the quoted passage, there should be no sign of incoherence.

Thomas Hurka also notices, albeit indirectly, that extrinsic value may enter into an account of organic unities as on the account I've proposed. Hurka points out, like I have above, that conditionalism requires the rejection of the view that intrinsic value supervenes on intrinsic properties. Hurka's response is that the definition of intrinsic value can be stretched so as to include the sort of value that an item may bear, conditional on features of the world that are external to it. On this, Hurka writes:

When it actually exists, conditional value plays the same role as uncontentiously intrinsic [i.e. supervenience-defined intrinsic] value. It contributes directly to the overall value of the world; it is something we should care about and pursue for its own sake because of its value. I therefore see no reason to rule out the looser definition of intrinsic value. ${ }^{24}$

The middle two sentences in this passage seem to indicate the same assumption that Dancy makes, namely, that parts are more fundamental, in the relevantly evaluative sense, than wholes they're in. At any rate, Hurka's claims that parts "contribute directly" and ought to be cared about for their "own sake" are puzzling if what is being allowed is that the value in question is coming from somewhere else. In fairness, Hurka is not concerned with making fully general claims about the nature of organic unities. He claims it would be "pedantry" to insist on restricting the definition of intrinsic value to its supervenience-oriented form.

Dancy and Hurka both overlook the possibility that an organic unity may occur because some amount of intrinsic whole-value may exist such that it can be identified as a result of noticing a certain amount of non-intrinsic part-value. At any rate, this possibility is what gets overlooked when one treats the 'contribution' of part-value as including the idea that part-value also makes it the case that there is the relevant amount of whole-value. A first advantage of the non-dispersion principle is that it allows an invariabilist to avoid commitment to this claim. The result is that an invariabilist can avoid one sort of objection to their view.

${ }^{24}$ (1998: 304). 
A different conditionalist objection to Moore is due to Christine Korsgaard. According to Korsgaard, invariabilist views leave out something important about the internal structure of organic unities. She argues that "Moore's view, and the intuitionistic method of isolation, veil or obscure the internal relations within the organic unity in virtue of which the organic unity has its value $^{, 25}$. Korsgaard appears to hold the view that whenever a whole or its parts may condition the values of the other, this rules out invariabilism about the intrinsic value of a whole's parts. This leads her to claim that Moore lacks the resources to make sense of these conditioning relations:

\begin{abstract}
On Moore's account the only relation in which the elements of an organic whole stand to each other is the relation of being elements in a single organic whole. They are all on a footing with one another. But if Kant is right there is an order within "valuable wholes," a conditioning of some elements by others, that is hidden by treating these elements as just so many ingredients. This order reflects the reason why the wholes are good. ${ }^{26}$
\end{abstract}

On my reading of this passage, Korsgaard's main point is that invariabilism makes no room for relations of evaluative priority within organic unities. Aimed at an invariabilist who relies solely on the relations principle, Korsgaard's objection might be accurate. (I shall later return to the question of whether it's right to think that all organic unities must be subject to any relations of priority.) However, this sort of "order within wholes" is precisely what the non-dispersion principle can accommodate. One way of summarizing my main theoretical point (in Korsgaardian terms) is like this: The intrinsic value of a whole may be affected by conditioning relations within that whole, even if these relations do not thereby affect the intrinsic value of the parts, but rather add extrinsic value (or maybe disvalue) to these parts. This is a possibility that Korsgaard's assessment of Moore overlooks.

As with Dancy, Korsgaard appears to assume that invariabilism includes the idea that intrinsic whole-value obtains in virtue of intrinsic part-value. This is what would make the parts "ingredients" of whole-value. In general, this is the sort of conditionalist opposition to Moore that invariabilists can overcome by endorsing the proposal I have made in this paper. If I am

\footnotetext{
${ }^{25}$ (1983: 271, italics original). When speaking of the "intuitionistic method of isolation", Korsgaard has in mind the familiar Moorean idea that intrinsic value supervenes on its bearer's intrinsic properties.

${ }^{26}$ (1983: 271).
} 
right, the possibility of adopting the non-dispersion principle shows that invariabilists are not committed to this. Invariabilism can accommodate ideas about "order within valuable wholes" because such "order" is about the conferral of extrinsic value. It therefore does not force the abandonment of Moorean ideas about intrinsic value and supervenience.

\section{What else might count against the relations principle?}

I have concentrated on explaining how the non-dispersion principle allows the invariabilist to overcome very general objections to invariabilism, made by influential conditionalist authors. Due to the general nature of these objections, I have discussed them without much attention to questions about which candidate organic unities are genuine and which are not. On the one hand, it may be an advantage of my proposals that they permit some neutrality about where to identify genuine cases of organic wholes. However, the non-dispersion principle might look more attractive if some sketch were given of how it actually works in an invariabilist account of particular organic unities. I shall use this final section to provide such a brief sketch.

Of course, invariabilists may disagree among themselves as to whether any candidate organic unity is a genuine one. Given this, I should say that the examples selected here are not intended to be ones that all invariabilists would accept as real organic unities. Rather, I intend to show how an invariabilist might give an account of these (putative) organic unities if they want to. As I remarked earlier in this paper, it is worth acknowledging that conditionalism may provide the correct account of some organic unities. Now, of course some invariabilists might want to argue that their view has very general application, and nothing I have to say prevents them from doing so. But I am not the first to suggest that a desire to see one account prevail as the sole general theory might be unmotivated ${ }^{27}$. In a similar spirit, one might allow that the relations principle provides a better invariabilist explanation of some organic unities than the non-dispersion principle can. Indeed, it might seem correct to say that part of what is intrinsically

\footnotetext{
${ }^{27}$ After drawing the distinction between invariabilism and conditionalism, Thomas Hurka suggests that "sometimes one and sometimes the other [might be] more appropriate for expressing a given evaluative view", and that it might be wrong-headed to suppose that there must be any "general superiority of [invariabilist] holism over conditionality" (1998: $300 \&$ 320). My points here are in the same spirit as Hurka's.
} 
valuable about a mosaic consists in certain spatial arrangements of stones whose respective intrinsic values add up to less than the mosaic's value. The explanation allowed by the nondispersion principle says, instead, that the mosaic confers extrinsic value on its stones. The intrinsic value of the mosaic can then be calculated by combining this extrinsic value with the stones' independent intrinsic values. I leave it open which explanation works best for the mosaic case, although it's worth noting that the non-dispersion principle might work at least as well as the relations principle here.

The non-dispersion principle seems to have a more obvious advantage over the relations principle in cases of malicious pleasure. As I have said, not everyone regards cases of malicious pleasure as an organic unity ${ }^{28}$. But since many people do, it is worth explaining why invariabilists might be best off with the non-dispersion principle when accounting for such cases. On any account that invokes the relations principle, the value of malicious pleasure consists in the positive value of pleasure, minus the disvalue of the relevant sadistic motives, along with some further adjustment taking into account the disvalue of some relation between these two. Including the disvalue of this relation shows how malicious pleasure is worse than the combination of unrelated pleasure and malice in distinct individuals. But it's not a very intuitive way of getting the job done. In particular, it's hard to resist the idea that malice in some way conditions pleasure. Pleasure borne out of malice plausibly has some of its value diminished, even if it undergoes no loss in intrinsic value ${ }^{29}$. The relations principle doesn't allow for this. It merely introduces some additional disvalue (that attaching to the relationship between pleasure and malice), leaving the value of the pleasure unmodified. It is this sort of problem that might encourage one to switch to a conditionalist account of malicious pleasures ${ }^{30}$.

\footnotetext{
${ }^{28}$ See for example Hurka (1998: 312). Hurka does regard undeserved pain as an organic unity. It is likely that my account would also explain this case better than an invariabilist view that used the relations principle. Zimmerman suggests that the lesson of malicious pleasures is that we cannot sensibly ask whether a pleasure is good until we know whether it is held maliciously (2001: 145). Compare Lemos (2006: 78-79). It is also worth remembering that intuitions about examples of malicious pleasure may be distorted by certain assumptions we make when confronting particular cases (Fletcher 2008). My point here doesn't rely on inferences drawn from any specific examples.

${ }^{29}$ Roderick Chisholm, for example, suggests that : We may say...that one pleasure is better than another pleasure if the intentional object of the one is better than the intentional object of the other; and that one displeasure is worse than another displeasure if the intentional object of the one is better than the intentional object of the other" (1986: 63). These remarks don't obviously select between conditionalism about value and the sort of invariabilism that might rely on ideas about non-dispersion of intrinsic value. But Chisholm's picture does, I think, count against relying on the relations principle.

${ }^{30}$ For more on this point, see Fletcher (2010: 166).
} 
However, if invariabilists adopt the non-dispersion principle then cases of malicious pleasure need not count in favour of any switch to conditionalism. Given the non-dispersion principle, the presence of a person's malice confers a certain amount of extrinsic disvalue on the pleasure gained due to (say) the satisfaction of sadistic desires. This pleasure is a part of a larger whole that combines that pleasure along with such sadistic desires. It is a part that has had value conferred on it by the whole it's in or, perhaps, by another part of this whole. The intrinsic value of the whole can thus be identified by an inference that makes reference to the extrinsic disvalue of the pleasure in question, as well as its intrinsic value (and the intrinsic disvalue, we might say, of the sadistic desires). This remains an invariabilist account, since the presence of malice doesn't affect the intrinsic value of the pleasure. Conditionalist accounts, by contrast, force upon us the claim that the mere sensation of pleasure is worth less when it occurs due to sadistic motives. Insofar as we wish to preserve the idea that intrinsic value can't be affected by items external to its bearer, we might prefer to say that a person's malice makes resulting pleasure extrinsically bad. More crudely, we might say that pleasure in a sadist is intrinsically good but happens to have something bad about it (perhaps non-intrinsically bad). Neither the relations principle nor conditionalism quite allows us to say this. But an appeal to the non-dispersion principle does.

It's worth pausing to note that the relations principle need not be wholly rejected in light of the above reasoning. For one thing, the coherence of that principle remains intact: There's nothing strange (in principle) about the idea of a relation between a whole's parts being itself a bearer of intrinsic value. What I've argued is that this idea can't give the invariabilist much of a response to certain conditionalist objections to invariabilism in general, and that it might not provide such an intuitive account of malicious pleasures as can be gained with the help of the non-dispersion principle. Nevertheless the invariabilist might seek to invoke the relations principle when accounting for the sorts of organic unities that don't, after all, seem subject to any internal conditioning of value, as in the mosaic case, perhaps. Conditionalists might not have this option of allowing that internal conditioning is something that obtains in only some organic wholes. Because conditionalists claim an organic unity obtains when a whole's parts undergo a change in intrinsic value, they must say that breaking up a mosaic would make its stones 
intrinsically worse. As I remarked at the outset, this seems rather odd. It's more intuitive to suggest that organic unities are sometimes subject to what Korsgaard calls "an order within wholes", and sometimes not. Insofar as invariabilists can invoke either the relations principle or the non-dispersion principle, they can quite plausibly accommodate this possibility.

Conditionalist opposition to Moore gets much of its traction from the apparent incompatibility of invariabilism about value with certain intuitive features of organic unites. I have argued that invariabilists can respond to conditionalist objections of this sort, and thereby overcome some worries about whether their position faces serious problems. I have not established any grander claim about whether it is invariabilism or conditionalism that offers the most plausible general account of organic unities. What I have said here might help invariabilists who wish to say that their position does have fully general application. But it also helps those who wish merely to say that invariabilist explanations have some application, whilst enhancing the invariabilist approach to certain organic unities in particular.

\section{Acknowledgements}

Earlier versions of this paper were presented to audiences at Stanford University and the 11th Conference of the International Society for Utilitarian Studies, held at the University of Pisa. I'd like to thank those who attended for their helpful feedback. For valuable conversations and/or comments on earlier drafts, I'd also like to thank: Ralf Bader, William Beals, John Broome, Joshua Cohen, Thomas Dougherty, Guy Fletcher, Nadeem Hussain, Krista Lawlor, and Gerard Vong. The paper was also helped by the comments of an anonymous referee for Philosophical Studies.

\section{References}

Bradley, B. (1998): “Extrinsic Value”, Philosophical Studies 91: 109-126

Bradley, B. (2002): “Is Intrinsic Value Conditional?” Philosophical Studies 107: 23-44

Bradley, B. (2006): “Two Concepts of Intrinsic Value” Ethical Theory and Moral Practice 9:

111-130 
Broome, J. (1991): Weighing Goods, Oxford: Blackwell

Brown, C. (2007): “Two Kinds of Holism about Values”, The Philosophical Quarterly 57/228: $456-463$

Chisholm, R. (1986): Brentano and Intrinsic Value, New York: Cambridge University Press

Dancy, J. (2003): “Are There Organic Unities?”, Ethics 113/3: 629-650

Dancy, J. (2004): Ethics Without Principles, New York: Oxford University Press

Dorsey, D. (2012): “Intrinsic Value and the Supervenience Principle" Philosophical Studies 157: 267-285

Fletcher, G. (2008): “The Consistency of Qualitative Hedonism and the Value of (at Least Some) Malicious Pleasures" Utilitas 20: 462-471

Fletcher, G. (2010): “Brown and Moore's Value Invariabilism vs Dancy’s Variabilism”, The Philosophical Quarterly 60/238: 162-168

Hurka, T. (1998): “Two Kinds of Organic Unity”, The Journal of Ethics 2: 299-320

Kagan, S. (1998): “Rethinking Intrinsic Value” The Journal of Ethics 2: 277-297

Korsgaard, C. (1983): “Two Distinctions in Goodness", reprinted in Creating the Kingdom of Ends, New York: Cambridge University Press

Langton, R. (2007): “Objective and Unconditioned Value”, The Philosophical Review 116: 157185

Lemos, N. (1994): Intrinsic Value: Concept and Warrant, New York: Cambridge University Press

Lemos, N. (2006): “Indeterminate Value, Basic Value, and Summation” in K.McDaniel, J.Raibley, R.Feldman \& M.Zimmerman (eds) The Good, the Right, Life and Death: Essays in Honor of Fred Feldman, Burlington, VT: Ashgate Publishing

Lewis, D. (1983): “Extrinsic Properties”, Philosophical Studies 44/2: 197-200

Rabinowicz, W., \& Ronnow-Rasmussen, T. (1999): “A Distinction in Value: Intrinsic and For Its Own Sake", Proceedings of the Aristotelian Society 100: 33-51

Zimmerman, M. (1999): "Virtual Intrinsic Value and the Principle of Organic Unities", Philosophy and Phenomenological Research 59: 653-666

Zimmerman, M. (2001): The Nature of Intrinsic Value, New York: Rowman \& Littlefield 